\title{
LE LIBRE CONSENTEMENT PRÉALABLE ET ÉCLAIRÉ
}

Contribution synthèse sur une pratique en développement

présenté par Véronique Lebuis

Montréal, juin 2009

C.-A. Poissant de recherche sur la gouvernance et I'aide au développement UQĀM

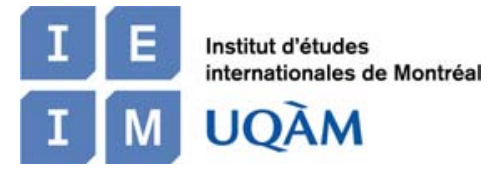


Le Libre consentement préalable et éclairé

Véronique Lebuis

LE LIBRE CONSENTEMENT PRÉALABLE ET ÉCLAIRÉ :

CONTRIBUTION SYNTHÈSE SUR UNE PRATIQUE EN DÉVELOPPEMENT

\section{VÉRONIQUE LEBUIS}

Juriste, détentrice d'une maîtrise en droit international et politique internationale de l'UQAM, chercheure au Groupe de recherche sur les activités minières en Afrique (GRAMA), UQAM, dans le cadre du projet Modes de gouvernance, institutions politiques et contributions du secteur minier au développement: leçons à partir de l'expérience canadienne.

http://www.grama.uqam.cal

UQÀM • Faculté de Science Politique et de Droit • Local A-1625

Téléphone (514) 987-3000 • Poste 2462

CP. 8888 - Succursale Centre-ville • Montréal (QC) • H3C 3P8 


\title{
LE LIBRE CONSENTEMENT PRÉALABLE ET ÉCLAIRÉ
}

\section{Contribution synthèse sur une pratique en développement}

\author{
Présenté par \\ Véronique Lebuis, chercheure GRAMA \\ Faculté de Science Politique et de Droit \\ Université du Québec à Montréal \\ juin 2009
}

Il est aujourd'hui largement admis que les peuples autochtones doivent obligatoirement être consultés lorsque des activités de développement sont susceptibles d'affecter leurs droits ${ }^{1}$. Le débat porte dorénavant sur le degré de contrôle qui devrait être accordé aux communautés locales et autochtones en ce qui concerne leur développement. L'évolution du principe selon lequel les communautés concernées devraient avoir le droit d'accorder ou de refuser leur libre consentement préalable et éclairé (LCPE) à la réalisation d'activités prenant place sur leurs terres ou ayant un impact sur les ressources dont ils dépendent illustre bien ce dilemme. En dépit des obstacles qui s'opposent à son développement, ce principe jouit d'une reconnaissance croissante sur le plan international comme moyen de mise en œuvre des droits des peuples autochtones et des communautés locales. Depuis son essor au début des années $1990^{2}$, son intégration croissante dans le droit des États, les normes internationales, ainsi que les standards et lignes directrices des meilleures pratiques volontaires dans le domaine du développement en ont fait une des préoccupations centrales des divers acteurs concernés ces enjeux. Néanmoins, bien que le principe de LCPE soit de mieux en mieux soutenu par les discours portant sur les droits humains et le développement, des questions substantielles demeurent quant aux meilleures façons de le mettre en œuvre.

Ces questions nous permettent de constater un paradoxe inhérent au principe du LCPE, renvoyant au processus même de sa conception. Celle-ci marque en effet de façon ambiguë une transition vers une remise en question des valeurs établies par et pour le maintien d'un système transnational et supranational qui se conjugue difficilement avec les réalités locales.

En cherchant à faire le point sur ce qui nous apparaît essentiel de considérer pour la suite des recherches dans ce domaine, nous élaborerons cette analyse en deux volets. Nous ferons d'abord un survol de la genèse du principe du LCPE, en nous attardant aux normes internationales, aux jurisprudences nationales et internationales et à l'évolution des codes

\footnotetext{
${ }^{1}$ Voir par exemple les décisions Nation Haïda c. Colombie-Britannique (Ministère des forêts), [2004] 3 R.C.S. 511 et Première Nation Tlingit de Taku River c. Colombie-Britannique (Directeur de l'évaluation de projet), [2004] 3 R.C.S. 550, sur lesquelles nous reviendrons plus loin.

${ }^{2}$ Les premières incarnations significatives du principe de LCPE s’inscrivant dans la Convention $n^{\circ} 169$ de l'Organisation internationale du travail en 1989.
} 


\section{Le Libre consentement préalable et éclairé Véronique Lebuis}

volontaires et des discours portant sur le développement durable. Ensuite, nous exposerons les enjeux et les débats soulevés par la mise en œuvre de ce principe. Ceci nous amènera enfin à dégager certains critères susceptibles de modeler la signification et les objectifs du principe du LCPE, en fonction des perceptions respectives des divers acteurs concernés. À cet effet, nous appuierons notre recherche sur la littérature reflétant la position des communautés - essentiellement à partir des études d'organismes nongouvernementaux (ONG) qui travaillent en coopération avec elles -, du Groupe de la Banque mondiale (GBM), des promoteurs de l'industrie, ainsi que des chercheurs qui s'intéressent au développement et à la mise en œuvre du principe de LCPE. Le lecteur trouvera également en annexe deux exemples de mise en application du principe du LCPE.

\section{I- Un principe issu d'une transition conflictuelle : genèse du principe de LCPE}

Les débats s'intensifient aujourd'hui au sujet du principe de LCPE dans plusieurs communautés locales et autochtones à travers le monde, ainsi que dans les salles de planification de nombreuses compagnies minières et pétrolières, de la Banque mondiale, de la Société financière internationale (SFI), comme au sein de plusieurs organes des Nations Unies.

L'origine du principe de LCPE se trouve dans le domaine médical, c'est-à-dire dans le cadre d'une relation individuelle entre patient et médecin visant, et vise à s'assurer que le médecin donne suffisamment d'information à son patient pour que ce dernier soit en mesure de prendre une décision éclairée dans le choix des traitements qui lui sont proposés $^{3}$. La transition du concept vers un dialogue de nature collective en accroît la complexité. D'abord reconnu comme essentiel à la reconnaissance et à la mise en œuvre de certains droits fondamentaux des peuples autochtones protégés par divers instruments de droit international, tels que le droit à l'autodétermination et au contrôle sur leur terres, territoires et ressources ${ }^{4}$, ce principe est devenu plus largement accepté comme partie intégrante d'un traitement juste et équitable pour toutes les communautés. C'est dans cette optique qu'en janvier 2005, sous l'égide du Forum permanent des Nations Unies sur les questions autochtones, plusieurs agences des Nations Unies se sont réunies dans le but d'évaluer leurs politiques et leurs pratiques liées au «Libre consentement préalable et éclairé $»^{5}$. Entre-temps, le Groupe des Nations Unies sur les populations autochtones,

\footnotetext{
3 Anne Perreault, «Facilitating Prior Informed Consent in the Context of Genetic Resources and Traditional Knowledge », Sustainable Development Law \& Policy, vol. 4, 2004, p. 21-26. Voir également Joshua P. Rosenthal, « Politics, Culture and Governance in the Development of Prior Informed Consent in Indigenous Communities », Current Anthropology, vol. 47, no. 1, février 2006, p. 119-142.

${ }^{4}$ En premier lieu les droits reconnus à l'article 1 commun au Pacte international relatif aux droits civils et politiques, 16 décembre 1966, (entrée en vigueur: 23 mars 1976, accession du Canada 19 mai 1976) et au Pacte international relatif aux droits économiques, sociaux et culturels, 16 décembre 1966, (entrée en vigueur : 3 janvier 1976, accession du Canada 19 mai 1976).

${ }^{5}$ C.S. Sena, «An Overview of a Current Understanding of FPIC as a Methodological Issue, in Activities Relating to Indigenous Peoples: Significance and Challenges », Statement to the International Expert
} 


\section{Le Libre consentement préalable et éclairé Véronique Lebuis}

dans l'exercice de son mandat relatif à la définition de standards sur les droits des peuples autochtones, travaillait à l'élaboration d'un document légal et de lignes directrices pour sa mise en œuvre ${ }^{6}$. Parmi d'autres organisations, les travaux de la Commission mondiale des barrages ont été déterminants dans la définition du principe de LCPE en tant que principe phare devant guider les étapes de construction de barrages qui pourraient affecter les peuples autochtones et les minorités ethniques ${ }^{7}$. De même, la Revue des industries extractives de la Banque Mondiale est arrivée à la conclusion que la reconnaissance et la mise en œuvre des droits des personnes affectées au consentement préalable et informé était une condition nécessaire à la réalisation de tout projet d'extraction afin d'assurer qu'il contribue à la réduction de la pauvreté et au développement durable, suivant les objectifs politiques du Groupe de la Banque mondiale ${ }^{8}$. En outre, le principe de LCPE figure sur les agendas de plusieurs organisations internationales telles que : la Convention sur la diversité biologique9, l'Organisation mondiale de la propriété intellectuelle (OMPI), ainsi que l'Organisation mondiale du commerce (OMC) en ce qui a trait à l'accès et au partage des bénéfices des ressources biologiques et des savoirs traditionnels associés. Par ailleurs, l'Union internationale pour la conservation de la nature (UICN), en lien avec l'établissement des parcs et des aires protégées, ainsi que d'autres banques multilatérales et agences de développement et de financement s'y attardent dans le cadre de la définition de leurs politiques de relocalisation et des lignes directrices d'autres projets qui affectent les peuples autochtones ${ }^{10}$.

\section{Normes internationales}

Le droit des peuples autochtones au à l'obtention du LCPE est reconnu dans une série d'instruments universels et régionaux de droits de la personne. L'Organisation internationale du travail (OIT) définit le principe de LCPE, dans le cadre de sa Convention $\mathrm{n}^{\circ} 169$ adoptée en 1989, comme le droit des communautés

Workshop on Methodologies Regarding Free, Prior and Informed Consent on Indigenous Peoples, United Nations Permanent Forum on Indigenous Issues, New York, 17-19 janvier 2005. Voir le rapport de cet atelier: Report of the International Workshop on Methodologies Regarding Free, Prior and Informed Consent and Indigenous Peoples, United Nations Permanent Forum on Indigenous Issues 2005, New York, 17-19 janvier 2005.

${ }^{6}$ Commission on Human Rights, Sub-Commission on the Promotion and Protection of Human Rights, Working Group on Indigenous Populations, Twenty-third session 18-22 July 2005, E/CN.4/Sub.2/AC.4/2005/WP.1.

${ }^{7}$ World Commission on Dams, « Dams and Development, A New Framework for Decision-Making », The Report of the World Commission on Dams, Earthscan Publications Ltd, London and Sterling, 2005.

${ }^{8}$ The World Bank Group Extractive Industries, The Final Report of the Extractive Industries Review, 3 août 2004, en ligne: site de la Société financière internationale http://www.ifc.org/ifcext/eir.nsf/AttachmentsByTitle/FinalResponse/\$FILE/EIRFinalResponse.pdf.

[Dernière consultation en date du 27 août 2008].

9 Convention sur la diversité biologique, Rio de Janeiro, 5 juin 1992. En ligne : http://www.cbd.int/doc/legal/cbd-un-fr.pdf.

${ }^{10}$ Jogi Cariño, «Indigenous Peoples' Right to Free, Prior, Informed Consent: Reflections on Concept and Practice », Arizona Journal of International \& Comparative Law, vol. 22, no.1, 2005, p. 19-39. 


\title{
Le Libre consentement préalable et éclairé Véronique Lebuis
}

\begin{abstract}
de décider de leurs propres priorités en ce qui concerne le processus de développement, dans la mesure où celui-ci a une incidence sur leur vie, leurs croyances, leurs institutions et leur bien-être spirituel et les terres qu'ils occupent ou utilisent d'une autre manière, et d'exercer autant que possible un contrôle sur leur développement économique, social et culturel propre. En outre, lesdits peuples doivent participer à l'élaboration à la mise en ouvre et à l'évaluation des plans et programmes de développement national et régional susceptibles de les toucher directement ${ }^{11}$.
\end{abstract}

Comme le rappelle Bernard Duhaime, lorsqu'un État ratifie la Convention no ${ }^{\circ} 169$, il s'engage envers les autres États et envers l'OIT à prendre les mesures nécessaires pour rendre celle-ci effective ${ }^{12}$. C'est en adoptant, sur le plan national, des lois et règlements qui peuvent résulter en obligations du gouvernement face aux citoyens, que l'État répond à ses obligations internationales, notamment en faisant respecter les règles qu'il impose aux entreprises en activité sur son territoire national. Cependant, suivant ce processus de mise en œuvre des obligations internationales, les peuples autochtones et locaux demeurent «dépendants des initiatives gouvernementales quant à l'intégration globale des normes et principes de la Convention à tous les aspects de la société civile $»^{13}$.

Dans la sphère des Nations Unies, le Comité pour l'élimination de la discrimination raciale enjoint pour sa part les États parties à la Convention ${ }^{14}$ à s'assurer que les membre des peuples autochtones ont un droit égal à la participation effective dans la vie publique et à ce qu'aucune décision affectant directement leurs droits et intérêts ne soit prise sans leur consentement informé ${ }^{15}$. Selon le comité, le droit au consentement informé est ainsi lié au droit de « prendre part au gouvernement et à la direction des affaires publiques » protégé par l'article 5(c) de la Convention ${ }^{16}$.

Les questions liées aux formes et à la nature de cette participation, cependant, demeurent ambigües. Quelles significations prennent les représentations des peuples autochtones et locaux pour l'industrie et les gouvernements responsables de faire appliquer les normes en matière de consultation ? En outre, quelles significations ces représentations prennentelles pour les populations effectivement représentées ? Comment savoir si elles parlent d'une seule voix et, dans le cas contraire, quel sort est réservé à la dissidence ? Autant de questions qui devraient se poser dans l'élaboration de chaque nouveau processus de consultation.

\footnotetext{
${ }^{11}$ Organisation international du Travail, Convention ( $\left.N^{\circ} 169\right)$ concernant les peuples indigènes et tribaux dans les pays indépendants, 1989 (entrée en vigueur: 5 septembre 1991).

12 Bernard Duhaime, «Évaluation des options à la portée des peuples autochtones pour veiller à l'application de la Convention 169 de l'OIT », janvier 2001. En ligne : http://www.ddrd.ca/site/publications/index.php?id=1329\&lang=fr\&subsection=catalogue [page consultée le 20 avril 2008].

13 Ibid.

${ }^{14}$ Comité pour l'élimination de la discrimination raciale, Convention internationale sur l'élimination de toutes les formes de discrimination raciale, Résolution 2106 A(XX), 21 décembre 1965, entrée en vigueur le 4 janvier 1969.

${ }^{15}$ General Recommendation XXIII (51) concerning Indigenous Peoples. Adoptée lors de la 1235e réunion du Comité, 18 août 1997. UN Doc. CERD/C/51/Misc.13/Rev.4, par. 3.

${ }^{16}$ Supra note 18 , art. 5(c).
} 


\section{Le Libre consentement préalable et éclairé Véronique Lebuis}

En 2001, le Comité des droits économiques, sociaux et culturels, créé par le Conseil économique et social des Nations Unies, constatait que les terres traditionnelles des peuples autochtones avaient été réduites ou occupées, sans leur consentement, par les compagnies forestières, minières et pétrolières, aux dépens de l'exercice de leur culture et de l'équilibre de l'écosystème. Le Comité recommandait par conséquent que les États parties au Pacte international relatif aux droits économiques, sociaux et culturels qu'ils " ensure the participation of indigenous peoples in decisions affecting their lives, (...) and seek the consent of the indigenous peoples concerned ${ }^{17}$. Ces remarques s'accordent avec l'approche adoptée par la Commission des Nations Unies pour les Corporations transnationales qui, après vingt ans de négociations, a produit en 1990 un code détaillé pour les multinationales qui stipule entre autres que :

les sociétés transnationales devraient respecter les objectifs sociaux et culturels, ainsi que les valeurs et traditions des pays où elles ouvrent [...] Les sociétés transnationales sont tenues de respecter les droits de la personne et les libertés fondamentales dans les pays où elles æuvvrent. Dans leurs relations sociales et industrielles, les sociétés transnationales sont tenues de ne pas exercer une discrimination en fonction de la race, de la couleur, du sexe, de la religion, de la langue, de l'origine sociale, nationale, ethnique ou politique ou de toute autre opinion ${ }^{18}$.

Bien que ce code n'ait jamais été adopté, il reflète sans contredit l'émergence d'une prise de conscience face aux responsabilités des compagnies transnationales en ce qui a trait aux aspects humains du développement.

\section{Jurisprudences nationales et internationales}

Parallèlement, les tribunaux font écho à ces normes en définition. Au cours des dix dernières années, un certain nombre de décisions judiciaires ont profondément modifié les relations existant entre les compagnies extractives et les communautés autochtones, en réitérant que les gouvernements avaient l'obligation de consulter et, le cas échéant, d'accommoder les communautés concernées avant le développement de projet affectant l'exercice des titres aborigènes ${ }^{19}$. Les systèmes régionaux de droits humains ont

17 Concluding Observations of the Committee on Economic, Social and Cultural Rights: Colombia. 30/11/2001. E/C.12/Add.1/74 par. 12 et 33, cité dans Forest Peoples Program et Tebtebba Foundation, $A$ Submission to the Special Representative of the Secretary-General on human rights and transnational corporations and other business enterprises, 29 décembre 2006. En ligne: http://www.businesshumanrights.org/Documents/Forest-Peoples-Tebtebba-submission-to-SRSG-re-indigenous-rights-29-Dec2006.pdf. [Dernière consultation le 22 mai 2008]

${ }^{18}$ Projet de Code de conduite pour les corporations transnationales, adopté le $1^{\mathrm{er}}$ février 1988, ONU. ESCOR, ONU Doc. E/1988/39/Add.1, 1988, paragraphes 13-14, dans Droits et démocratie, Au-delà des engagements : l'action - De la Conférence mondiale des Nations Unies sur les Droits de l'homme à la Conférence des Nations Unies sur le financement $d u$ développement, 15 mars 2002. En ligne: http://www.dd-rd.ca/site/publications/index.php?id=1533\&lang=fr\&page=4\&subsection=catalogue. [Dernière consultation le 22 mai 2008].

19 Au Canada, la première décision significative en ce sens est l'affaire Haïda (supra note 1), qui, en rétablissant en partie la nature d'une relation de nation à nation entre les peuples autochtones et les gouvernements, démontre l'importance de la structure des institutions consultatives en vue de la 


\section{Le Libre consentement préalable et éclairé Véronique Lebuis}

également soutenu le droit des peuples autochtones au principe de LCPE sur l'utilisation de leurs terres et de leurs ressources. La Commission interaméricaine des droits de l'homme (CIDH) a conclu que le droit interaméricain exigeait [des] « special measures to ensure recognition of the collective and particular interest that indigenous peoples have in the occupation and use of their traditional lands and resources and the right not to be deprived of this interest except with fully informed consent, under conditions of equality, and with fair compensation $»^{20}$. En corollaire, la proposition de Déclaration interaméricaine sur les droits des peuples autochtones, élaborée par l'Organisation des états américains (OÉA), prévoit que les États devraient obtenir leur consentement préalablement à l'approbation de tout projet affectant leurs terres, territoires et ressources; et cela particulièrement en ce qui concerne le développement, l'utilisation ou l'exploration des ressources naturelles ${ }^{21}$. La Cour interaméricaine des droits de l'homme a appliqué ce principe dans l'affaire Saramaka en reconnaissant que le devoir dévolu à l'État de consulter le peuple Saramaka dès la proposition d'un projet de développement doit avoir pour objectif d'en arriver à une entente, ce qui requiert de l'État à la fois de recevoir et de diffuser l'information pertinente dans une forme compréhensible et accessible au public. De plus, la Cour précise qu'en fonction du niveau d'impact du projet proposé, l'État peut se voir exiger d'obtenir le libre consentement préalable et éclairé en accord avec les coutumes et traditions du peuple Saramaka ${ }^{22}$. Dans la même

conciliation des souverainetés respectives. Même si en dernière instance ce jugement relève de la légitimation de l'affirmation de la souveraineté canadienne sur les Autochtones (voir notamment l'analyse de Boisselle, infra note 38), il pose néanmoins les premiers jalons d'une recherche de dialogue entre des identités mutuellement reconnues.

${ }^{20}$ Inter-American Commission on Human Rights, Report No. 75/02, Case No. II.140, Mary and Carrie Dann (United States), 27 décembre 2002, OEA/Ser.L/V/II.116, doc. 46, par. 131. Voir aussi Report No. 96/03, Maya Indigenous Communities and their Members (Case 12.053 Belize), 24 octobre 2003, par. 116.

${ }^{21}$ Steven Herz, Antonio La Vina, Jonathan Sohn, « Development without conflict. The Business Case for Community Consent $»$, World Resources Institute, mai 2007, p.14.

${ }^{22}$ Cf. Case of the Saramaka People v. Suriname. Preliminary Objections, Merits, Reparations, and Costs. 28 novembre 2007. Serie C no. 172, par. 133 à 137. Le jugement de la Cour en ce qui a trait aux coutumes et traditions exige que l'État consulte le peuple Saramaka « regarding at least the following six issues: (1) the process of delimiting, demarcating and granting collective title over the territory of the Saramaka people (para. 194(a)); (2) the process of granting the members of the Saramaka people legal recognition of their collective juridical capacity, pertaining to the community to which they belong (para. 174); (3) the process of adopting legislative, administrative, and other measures as may be required to recognize, protect, guarantee, and give legal effect to the right of the members of the Saramaka people to the territory they have traditionally used and occupied (para. 194 (c)); (4) the process of adopting legislative, administrative and other measures necessary to recognize and ensure the right of the Saramaka people to be effectively consulted, in accordance with their traditions and customs (para. 194 (d)); (5) regarding the results of prior environmental and social impact assessments (para. 133), and (6) regarding any proposed restrictions of the Saramaka people's property rights, particularly regarding proposed development or investment plans in or affecting Saramaka territory (para. 129)». De plus «[the] Court deliberately omitted from the Judgment any specific consideration as to who must be consulted. By declaring that the consultation must take place "in conformity with their customs and tradition", the Court recognized that it is the Saramaka people, not the State, who must decide which person or group of persons will represent the Saramaka people in each consultation process ordered by the Tribunal (para. 133) ». Voir Inter-American Court of Human Rights, Case of the Saramaka People v. Suriname, 12 août 2008 (Interpretation of the Judgment on Preliminary Objections, Merits, Reparations, and Costs), Series C No.185. 


\section{Le Libre consentement préalable et éclairé Véronique Lebuis}

veine, la Commission européenne des droits de l'homme a reconnu le droit des peuples autochtones de « s'objecter aux projets », ce qui comprend le droit au LCPE ${ }^{23}$.

\section{Codes volontaires}

Le principe du LCPE pour les peuples autochtones a également été reconnu dans plusieurs processus d'établissement de standards volontaires élaborés selon les «meilleures pratiques » des industries ayant un haut potentiel d'impact spécifique ${ }^{24}$. Par exemple, le Forest Stewardship Council, une collaboration multisectorielle conçue pour établir des normes pour l'industrie forestière, reconnaît que les peuples autochtones ont le droit de contrôler les ressources forestières sur leurs terres, à moins d'en déléguer le contrôle selon leur libre consentement éclairé ${ }^{25}$. La Commission mondiale sur les barrages a également reconnu l'importance de respecter le droit des communautés autochtones à consentir aux activités susceptibles d'avoir un impact sur leurs terres et ressources $^{26}$. De même, la Revue des industries extractives de la Banque Mondiale, une étude indépendante portant sur les impacts du développement promu par le Groupe de la Banque mondiale (GBM) dans les secteurs du pétrole, des mines et du gaz, approuve le principe du LCPE pour les peuples autochtones ${ }^{27}$. Cependant, tel que nous le verrons plus loin, le GBM a décidé d'adopter une position significativement différente sur la portée à donner à ce principe dans le cadre de la mise en œuvre de ses politiques de financement de projets de développement.

Tous ces instruments évoquent en somme le droit des peuples autochtones de déterminer les priorités de développement de leurs terres, territoires et ressources. La Convention sur la diversité biologique, qui n'est pas à proprement parler un instrument de protection des droits de la personne, traite également du principe du LCPE en reconnaissant la nécessité de la participation des populations autochtones, en fonction de leurs connaissances respectives, aux prises de décisions relatives à des activités de développement affectant leurs territoires traditionnels et les ressources nécessaires à la préservation de leur mode de vie. Dans la pratique, cette reconnaissance soulève l'épineuse question de savoir si les terres, territoires et ressources appartiennent légalement aux peuples concernés et quelle est la portée de leur pouvoir décisionnel eu égard à leurs titres de propriété éventuels.

\footnotetext{
${ }^{23}$ Steven Herz et al., op. cit., p. 15.

${ }^{24}$ Certains auteurs font toutefois remarquer qu'il ne faut pas nécessairement se fier à des standards de types « meilleures pratiques » sans au préalable avoir établi des critères sérieusement documentés et spécifiques aux milieux et projets sur lesquels ces standards sont fondés. Voir en ce sens Melissa Marschke, David Szablowski et Peter Vandergeest, «Indigenous Peoples Scoping Exercise. Synthesis Report», Working Paper 21 of the Rural Poverty and Environment Working Paper Series, Centre de Recherche en Développement international (CRDI), janvier 2007, p. 30.

${ }^{25}$ Marcus Colchester et Maurizio Farhan Ferrari, « Making FPIC, Free, Prior and Informed Consent, Work: Challenges and Prospects for Indigenous Peoples », Forest Peoples Programme, juin 2007, p. 16.

${ }^{26}$ Supra note 7.

${ }^{27}$ Supra note 8, p. 18.
} 


\section{Le Libre consentement préalable et éclairé Véronique Lebuis}

L'exemple des cours d'eau illustre bien la complexité des défis qui attendent les différentes parties prenantes aux négociations, dans la mesure où les répercussions d'un projet peuvent se faire sentir sur des communautés qui n'ont pas le «contrôle légal $»^{28}$ des territoires où le projet se déroule; celui-ci pourrait même se trouver dans un autre pays.

\section{$\underline{\text { II- Enjeux et débats }}$}

En abordant la question du consentement des communautés autochtones, les diverses parties prenantes à l'élaboration d'un projet de développement doivent pouvoir en anticiper, dans une mesure raisonnable, les impacts positifs et négatifs. Même lorsque les parties admettent que les principes de consultation et de participation des populations affectées doivent guider les processus de négociations, elles butent encore sur le degré décisionnel que représente le droit au consentement, qui est fréquemment associé à un droit de veto sur la destinée d'un projet. Le débat entre l'attribution d'un droit de consultation ou un droit au consentement laisse place à un éventail d'approches et de normes plus ou moins contraignantes.

La distinction entre les termes de consultation et de consentement réside essentiellement, dans le fait qu'un «droit au consentement» signifie que la possibilité que les projets puissent être rejetés doit être connue par les communautés concernées et reconnue par les autres parties prenantes à la proposition de développement. Les réticences des gouvernements envers le principe de LCPE relèvent généralement, en effet, du droit de veto que le «droit au consentement» serait susceptible d'accorder aux populations visées. En ce sens, accorder un droit de veto à un groupe de la population nationale pourrait être interprété comme une restriction indue de la souveraineté sur laquelle repose la légitimité de l'État; de plus, il pose la question de la hiérarchisation de l'intérêt national et local.

La recherche d'un équilibre entre les souverainetés respectives représente en effet un enjeu majeur des négociations relatives au développement des ressources locales. Il constitue d'ailleurs le talon d'Achille de la mise en œuvre du LCPE en ce qu'il tend fréquemment à opposer plutôt qu'à rendre complémentaire l'intérêt national à l'intérêt local. En conséquence, certains gouvernements considèrent qu'octroyer à une petite partie de sa population nationale un droit décisionnel sur un projet susceptible de faire fructifier l'économie du pays revient à hypothéquer son pouvoir souverain sur les richesses naturelles de l'État. Or, tel que le faisait déjà remarquer la Cour internationale de justice en 1996,

\footnotetext{
${ }^{28}$ En ce sens si les lois relatives à la propriété et à la souveraineté territoriales sont nécessaires au fonctionnement global, elles peuvent représenter un obstacle de taille à la pleine participation des populations effectivement affectées dans le cadre d'un projet donné. Les instruments de droit international et transnational s'occupent ainsi de régler les différends entre États, mais les populations se trouvent généralement hors de la portée de ces instruments.
} 


\section{Le Libre consentement préalable et éclairé Véronique Lebuis}

In its ongoing development, the concept of human rights has long passed the stage when it was a narrow parochial concern between sovereign and subject. We have reached the stage, today, at which the human rights of anyone, anywhere, are the concern of everyone, everywhere. The world's most powerful States are bound to recognize them, equally with the weakest, and there is not even the semblance of a suggestion in contemporary international law that [human rights] obligations amount to a derogation of sovereignty ${ }^{29}$.

Pour les communautés, il peut y avoir problème dans la mesure où 1) les consultations sont organisées précisément avec l'objectif d'inciter au consentement plutôt que de proposer un libre choix, et 2) la détermination de l'autorité décisionnelle à travers laquelle s'exprime le consentement pose difficulté.

Cela conduit nécessairement à se questionner sur la légitimité des processus de consultation mis en place. David Szablowski pose en termes de "right process » la problématique de légitimation de l'autorité légale qui peut trouver appui sur différents modèles qu'il regroupe en quatre catégories: 1) la prise de décision par l'autorité appropriée, 2) l'autonomie des prises de décisions dans le domaine privé, 3) la participation publique aux prises de décisions et 4) la prise de décision par des experts ${ }^{30}$. En accordant un pouvoir décisionnel directement aux communautés afin de tenir compte de leurs priorités, le modèle participatif demeure à définir en fonction de chaque situation, puisque l'expression du LCPE est tributaire de différents facteurs, notamment culturels, propres à chaque groupe concerné.

Pour sa part, le rapport du World Resources Institute (WRI) distingue ces deux termes de la manière suivante: «[LCPE] differs importantly from mere consultation in the way decision-making authority is exercised and legitimated ${ }^{31}$. La consultation n'implique pas de partager ou de transférer l'autorité décisionnelle à ceux qui seront directement affectés par la décision. De plus, les mécanismes de consultation ne facilitent pas nécessairement une prise de décision plus inclusive ou collaborative, et constituent rarement une forme d'engagement du public visant à lui conférer du pouvoir ${ }^{32}$. À l'inverse, le principe de LCPE permettrait aux communautés concernées de participer significativement au processus de prise de décision, de négocier des avantages justes et propres à lier leurs vis-à-vis aux négociations, ainsi que de refuser leur consentement si leurs besoins, priorités et préoccupations ne sont pas adéquatement discutés. Les communautés auraient ainsi un meilleur contrôle sur les retombées d'un projet.

\footnotetext{
${ }^{29}$ Application of the Convention on the Prevention and Punishment of the Crime of Genocide (Bosnia and Herzegovina v. Yugoslavia (Serbia and Montenegro)), Separate Opinion of Judge Weeramantry, 11 juillet 1996, p. 56. En ligne: Cour internationale de justice, http://www.icj-cij.org/docket/files/91/7360.pdf [dernière consultation le 21 mai 2008].

${ }^{30}$ David Szablowski, Transnational Law and Local Struggles. Mining, Comunities and the World Bank, Hart Publishing, Oxford et Portland, 2007. Voir le chapitre I.

${ }^{31}$ Supra note 21, p.14.

${ }^{32}$ Voir World Bank, The World Bank and Participation, Washington, DC, 1994.
} 


\section{Le Libre consentement préalable et éclairé Véronique Lebuis}

Au Canada, l'idée d'institution consultative a ainsi tranquillement fait son chemin en passant par les parcours judiciaires. Graduellement, à partir de l'arrêt Guérin ${ }^{33}$ en 1984, en passant par l'arrêt Sparrow ${ }^{34}$ en 1990, la trilogie Van der Peet ${ }^{35}$, Gladstone $^{36}$ et Smokehouse $^{37}$ en 1996, la Cour suprême a accepté que l'idée d'égalité est nécessaire au succès de la consultation des Premières Nations, dans la perspective d'un mécanisme approprié pour rejoindre la réconciliation entre nations, soit la négociation de traités ${ }^{38}$. Pourtant, les juges persistent à ne considérer qu'une caractérisation étroite de la notion de droits ancestraux qui serait cohérente avec l'autonomie gouvernementale et le droit de s'autodéterminer. Dans cette optique, la Cour a statué dans l'affaire Delgamuukw ${ }^{39}$, en 1997, que lorsqu'il s'agit du droit au territoire lui-même, qui «comprend le droit de choisir les utilisations qui peuvent [en] être faites $\gg{ }^{40}$, l'obligation de consulter prend une dimension telle que pour porter atteinte au titre aborigène d'une manière qui respecte le rapport fiduciaire qui la lie aux autochtones, la Couronne doit faire "participer les peuples autochtones à la prise de décisions concernant leurs terres ${ }^{41}$, ce qui implique, selon la Cour, qu'il y a toujours obligation de consultation ${ }^{42}$. Selon la Cour, dans tous les cas la consultation doit être menée de bonne foi, dans l'intention de tenir compte réellement des préoccupations des peuples autochtones dont les terres sont en jeu. Certaines situations pourraient même exiger l'obtention du consentement d'une nation autochtone, particulièrement lorsque les provinces adoptent des règlements de chasse et de pêche visant des territoires autochtones ${ }^{43}$.

Lawrence et Macklem montrent pour leur part que le processus consultatif doit donner lieu à de réelles négociations sur les droits en cause et les attentes acceptables ${ }^{44}$. Selon eux, la négociation est en effet mieux adaptée à la résolution des multiples aspects des différends territoriaux entre la Couronne et les Autochtones, et produit des résultats plus légitimes puisque ce sont les parties elles-mêmes qui collaborent «directly and

\footnotetext{
${ }^{33}$ Guérin c. La Reine, [1984] 2 R.C.S. 335.

${ }^{34}$ R. c. Sparrow, [1990] 1 R.C.S. 1075.

${ }^{35}$ R. c. Van der Peet, [1996] 2 R.C.S. 507.

${ }^{36}$ R. c. Gladstone, [1996] 2 R.C.S. 723.

${ }^{37}$ R. c. N.T.C. Smokehouse Ltd., [1996] 2 R.C.S. 672.

${ }^{38}$ Andrée Boisselle, « De la consultation des peuples autochtones : Structure institutionnelle d'un dialogue appelé à renouveler la notion des droits ancestraux. Essai fondé sur la jurisprudence de la Cour suprême du Canada (1984-2004) et la théorie du droit », Mémoire de maîtrise en droit, Faculté de droit de l'Université de Montréal, 2006.

${ }^{39}$ Delgamuukw c. Colombie-Britannique, [1997] 3 R.C.S. 1010.

${ }^{40}$ Ibid. au par. 168.

${ }^{41}$ Ibid.

${ }^{42}$ Ibid.

${ }^{43}$ Ibid.

${ }^{44}$ Sonia Lawrence et Patrick Macklem, « From Consultation to Reconciliation: Aboriginal Rights and the Crown's Duty to Consult », Revue canadienne du Barreau, no. 79, 2000.
} 


\section{Le Libre consentement préalable et éclairé Véronique Lebuis}

constructively in fashioning a complex set of mutually agreeable trade-offs $\rangle^{45}$. La Cour parle même dans l'affaire Hä̈da d'un processus de négociation honnête " [qui] permet de concrétiser [la promesse contenue dans l'article 35 de la Loi constitutionnelle de 1982] et de concilier les revendications de souveraineté respectives " ${ }^{46}$. La Cour y amorce en ce sens une description de ce que devrait être la structure institutionnelle d'un processus de consultation axé sur la reconnaissance mutuelle, tout en spécifiant qu' "il n'y a pas obligation de parvenir à une entente mais plutôt de procéder à de véritables consultations» et que les demandeurs autochtones «ne devraient pas défendre des positions déraisonnables pour empêcher le gouvernement de prendre des décisions ou d'agir dans les cas où, malgré une véritable consultation, on ne parvient pas à s'entendre [...] Toutefois, le seul fait de négocier de façon serrée ne porte pas atteinte au droit des Autochtones d'être consultés ». Finalement, «[l']élément central n'est pas le résultat, mais le processus de consultation et d'accommodement $\gg{ }^{47}$.

\section{Signification du concept et conditions de mise en ouvre: différentes perspectives vers une définition commune du principe de LCPE?}

Pour Robert Walker, vice-président du Sustainability for Ethical Funds, l'histoire récente présente de nombreux cas de compagnies minières et pétrolières d'envergure ayant vu leur investissement s'évaporer, leur réputation salie et leur part de marché s'effondrer en raison de leur négligence à informer et à consulter correctement les communautés autochtones $^{48}$. Selon lui c'est ce qui oblige aujourd'hui les compagnies à développer de nouvelles approches face aux communautés et à considérer l'adoption des standards du principe de LCPE afin de s'assurer un retour sur leur investissement massif. Mais quels sont précisément ces «standards »? À la lumière de ce que nous venons d'exposer, l'idée d'un mécanisme standard de certification attestant de la validité des consultations entreprises et de la légitimité des ententes obtenues paraît assurément abstraite ${ }^{49}$. Chaque consultation prenant place dans un contexte unique, subissant les multiples aléas inhérents à la construction d'un cadre de discussion entre de nouveaux partenaires aux valeurs et intérêts distincts, il est permis de penser que l'établissement d'une procédure commune du LCPE pourrait escamoter l'esprit même d'un principe visant à tenir compte des réalités de chacun des intervenants à l'entente. Certaines expériences d'un processus de certification ont d'ailleurs démontré qu'il était possible qu'un certificat soit émis en

\footnotetext{
${ }^{45}$ Ibid., p. 279.

${ }^{46}$ Nation Haïda c. Colombie-Britannique (Ministère des forêts), op. cit., par. 20.

${ }^{47}$ Ibid., aux par. 42, 46 et 63.

${ }^{48}$ Canadian Resource companies must adopt new standards on community engagement, communiqué de presse, Vancouver, 5 mars 2008, en ligne: site de Ethical funds, www.ethicalfunds.com. [Dernière consultation en date du 28 avril 2008].

${ }^{49}$ L'établissement d'un processus obligatoire commun de consultation a fait l'objet de débat; voir par exemple le "Mining Certification Evaluation Project», dernière mise à jour juillet 2006, en ligne : http://www.minerals.csiro.au/sd/SD MCEP.htm. La problématique de la certification a également été abordée avec habileté par Szablowski, op. cit., p. 291.
} 


\section{Le Libre consentement préalable et éclairé Véronique Lebuis}

faveur d'une compagnie en dépit du dépôt de plaintes officielles à propos de certaines procédures par les populations concernées ${ }^{50}$.

Par contre, s'il faut reconnaître qu'il n'en existe aucune définition complète et universelle, la signification du principe LCPE se définit progressivement dans des arènes où jouent des intérêts variés.

En septembre 2007, l'Assemblée générale des Nations Unies adoptait la Déclaration sur les droits des peuples autochtones ${ }^{51}$, qui reconnaît l'obligation des États de consulter les peuples autochtones et d'obtenir leur « consentement préalable, donné librement et en connaissance de cause » avant d'adopter et d'appliquer des mesures législatives ou administratives susceptibles de les concerner ${ }^{52}$. Bien que la Déclaration n'ait pas force de loi, elle n'en exprime pas moins un large consensus international, qui se reflète également dans les standards et dans les lignes directrices produits par des groupes du secteur de l'industrie, s'appliquant également aux peuples locaux non-autochtones. Dans leur recherche d'une "licence sociale» pour opérer, les promoteurs des investissements doivent passer par un processus leur permettant de gagner la confiance des populations affectées. La «participation» des communautés constitue une part essentielle de ce processus et s'établit en tant qu'élément standard de la responsabilité sociale des entreprises. Le GBM fait partie des institutions qui requièrent une «participation significative », impliquant de parvenir à des ententes sur les mesures de précaution, de mitigation et de compensation ${ }^{53}$. Le partage des bénéfices entre les promoteurs, les gouvernements local et central, et les communautés affectées, représente aussi un élément essentiel du principe de participation significative. Selon Goodland, une "participation significative » adéquatement mise en œuvre devrait conduire au respect du principe de LCPE. Or, l'expression de "participation significative » se prête à des interprétations variées, en fonction de qui est appelé à gérer cette participation. En effet, si le GBM exige aujourd'hui la mise en œuvre d'une participation significative, le consentement ne fait pas partie des pré-requis à l'aval qu'il donnera à un projet ${ }^{54}$. Par ailleurs, comme l'explique Maxime St-Hilaire, la définition des droits qui peuvent être reconnus aux peuples autochtones au terme d'un processus de négociation avec l'État se heurte au fait

\footnotetext{
50 Tebtebba Foundation, «Statement: Recent experiences and recommendations on the concept and implementation of the principle of free, prior and informed consent », 15-26 mai 2006, en ligne: site du Southern African Regional Poverty Network, http://www.sarpn.org.za/documents/d0002040/index.php.

${ }^{51} \mathrm{~A} / \mathrm{RES} / 61 / 295 \mathrm{du} 13$ septembre 2007.

${ }^{52}$ Ibid., article 19.

${ }^{53}$ Robert Goodland, « Free, Prior and Informed Consent and the World Bank Group », Development Law \& Policy, vol. 4, 2004, p. 66.

${ }^{54} \mathrm{Au}$ sujet de la politique opérationnelle 4.10 sur les peuples autochtones, voir par exemple Goodland, idem., et Fergus MacKay, «The draft World Bank Operational Policy 4.10 on Indigenous Peoples: Progress or more of the same? »(2005), Arizona Journal of International \& Comparative Law, vol. 22, 2005, p. 65-98.
} 


\section{Le Libre consentement préalable et éclairé Véronique Lebuis}

que «dans l'esprit des agents de l'État, les autochtones ne peuvent avoir pour droits spéciaux que ceux que le droit posé par ce même État veut bien leur octroyer ${ }^{55}$.

Le rapport du WRI, reflétant la perspective du secteur industriel, identifie six principes d'importance cruciale dans la définition et la mise en œuvre des procédures de consentement, qui rejoint la perspective de nombreuses autres institutions gouvernementales et organisations non-gouvernementales en matière de LCPE :

- L'information relative au projet proposé doit être suffisante et transmise dans un langage accessible aux communautés concernées, tout en allouant le temps approprié pour que les communauté révisent et discutent des informations reçues avant d'engager une décision,

- L'inclusion des tous les membres de la communauté intéressés et encouragés à participer au processus de LCPE, incluant les parties prenantes affectées par des impacts indirects ou cumulatifs,

- Le dialogue doit être formalisé et continu à travers toutes les étapes du projet,

- La reconnaissance légale des accords négociés dans le cadre d'un processus de LCPE,

- Le suivi et l'évaluation doivent faire l'objet de mécanismes indépendants afin notamment de s'assurer que les plaintes des membres de la communauté concernées puissent être entendues,

- La reconnaissance, pour la compagnie, du principe de LCPE en tant que coût inhérent et nécessaire du projet de développement. ${ }^{56}$

En outre, si le principe de LCPE apparaît de plus en plus comme un repère légal pour les communautés locales, et autochtones en particulier, il fait donc en contrepartie office de ligne directrice pour les compagnies. Le AA1000 Stakeholder Engagement Standard (AA1000SES) est à ce titre un exemple de tentative des promoteurs du développement de réunir de meilleures pratiques de multiples organisations en une formule systématique permettant d'améliorer le niveau d'engagement de l'industrie envers sa responsabilité sociale $^{57}$. Promus par l'Organisation des Nations Unies, les Objectifs du Millénaire pour

\footnotetext{
${ }^{55}$ Maxime St-Hilaire est doctorant de l'Institut de droit comparé, Université McGill; auteur d'un mémoire de maîtrise sur la politique de continuité du gouvernement Charest relative aux revendications territoriales autochtones. Voir «Politique de continuité du gouvernement Charest - Vers une nouvelle génération de traités avec les autochtones? », Le Devoir, mardi 15 juillet 2003.

${ }^{56}$ WRI Report, supra note 21 à partir de la p.12.

57 Voir par exemple Ethical Corporation, en ligne : http://www.ethicalcorp.com/content.asp? ContentID=3970.
} 


\section{Le Libre consentement préalable et éclairé Véronique Lebuis}

le développement $(\mathrm{OMD})^{58}$, qui font office de plan directeur pour la réduction de la pauvreté et pour une amélioration des conditions de santé et d'éducation, constituent par ailleurs une référence pour quelques compagnies qui peuvent jouer un rôle déterminant en alignant leur projet de développement avec les besoins et les aspirations des communautés.

Andrée Boisselle analyse les principes structurels d'une consultation légitime des peuples autochtones, qu'elle fonde notamment sur la théorie interactive de Lon Fuller ${ }^{59}$. Elle démontre l'importance d'une égalité "de statut» entre les parties (autochtones et gouvernementales) au processus consultatif. L'analogie avec le principe de LCPE semble appropriée dans la mesure où cette égalité de statut devrait, d'après Boisselle, faire en sorte que «peu importe les moyens respectifs des adversaires, les mécanismes mis en place leur donnent à chacun une chance égale non seulement de parler (de présenter adéquatement son point de vue et d'interroger l'autre sur le sien), mais d'être entendus $»{ }^{60}$. En fonction de ces exigences structurelles relatives au processus consultatif, les critères d'égalité qu'elle estime nécessaire sont : 1) l'accès au processus, 2) la réponse aux préoccupations exprimées (être écouté), 3) la présence d'un tiers ${ }^{61}$, 4) la «culture » du processus (incluant la langue des échanges, la considération des savoirs traditionnels autochtones et de leurs systèmes de prise de décision). Pour y parvenir les autochtones doivent être en mesure de participer dès le stade de la conception du processus et les solutions, objet d'un processus continu de discussion, sont susceptibles d'être constamment modifiées suivant les actions et réactions des participants. Elle traite également des éléments structurels qui, selon elle, sont susceptibles d'influencer positivement l'attitude des parties, déterminante de la conduite du processus consultatif. Elle sépare ensuite en deux étapes le processus de dialogue, la première visant " l'apprentissage des points de vue et de l'identité des autres », la seconde étant consacrée à l'atteinte des objectifs stratégiques de la consultation. Puisque la conciliation des intérêts respectifs ne se déroule ainsi qu'en un deuxième temps, on devrait selon elle songer à axer le processus consultatif avant tout sur le dialogue «non stratégique », tout en tenant compte du facteur temps qu'elle considère " indispensable à la construction d'une relation de confiance, elle-même essentielle pour prendre des décisions de gouvernance légitimes ", et dont on pourrait tenir compte en ajoutant un principe de permanence à la structure de consultation ${ }^{62}$.

\footnotetext{
${ }^{58}$ Voir en ligne : http://www.un.org/french/millenniumgoals/.

${ }^{59}$ Dont L.L. Fuller, « Means and Ends » dans K. Winston, (dir.), The Principles of Social Order - Selected Essays of Lon L. Fuller, édition révisée, Hart Publishing, Oxford et Portland, 2001, p. 61.

${ }^{60}$ Boisselle, op. cit., p. 114.

${ }^{61}$ Il est intéressant de considérer cette exigence d'égalité en fonction de la présence d'un tiers «neutre », puisque cette présence n'est, à notre connaissance, généralement pas requise par les mécanismes de négociation entre les diverses parties prenantes sur les enjeux de développement. À cet effet, Fuller relève que « the group of two, the dyad, is peculiarly handicapped in resolving problems of internal order ». L.L. Fuller, « Mediation - Its Forms and Functions » dans K. Winston, dir., The Principles of Social Order Selected Essays of Lon L. Fuller, édition révisée, Hart Publishing, Oxford - Portland, 2001, p. 141, cité par Boisselle, p. 120.

${ }^{62}$ Boisselle, op. cit., p. 129-131.
} 


\section{Le Libre consentement préalable et éclairé}

\section{Véronique Lebuis}

On pourrait donc conclure que les meilleures pratiques en matière de développement consistent en ce qu'une communauté livre son consentement librement et sans coercition, préalablement à toute décision significative relative à un projet de développement, en ayant été pleinement informée grâce à un accès véritable à une information adéquate et complète sur les aspects économiques, sociaux et environnementaux du projet proposé. Les promoteurs du projet lui ont de plus alloué un temps suffisant et un accès aux expertises techniques nécessaires à l'acquisition d'une compréhension complète des impacts et des bénéfices du projet, tout en admettant ouvertement que la communauté n'avait pas l'obligation d'accorder son consentement. Enfin, le consentement a été exprimé par les représentants légitimes de la communauté, suivant un processus décisionnel conforme aux pratiques et significations culturelles de la communauté. 


\section{Le Libre consentement préalable et éclairé \\ Véronique Lebuis}

\section{$\underline{\text { Annexe : exemples de mise en ouvre }}$}

Suivant leurs engagements internationaux, en vertu de diverses conventions et traités internationaux, certains États modifient leurs lois nationales pour y inclure de nouvelles dispositions visant à protéger les droits des peuples autochtones. Hormis un apparent souci pour une plus ample participation des communautés affectées au partage des bénéfices découlant des projets de développement des ressources naturelles - bénéfices liés au partage des redevances, à la création d'emplois, à l'amélioration des systèmes d'éducation et des services de base, au développement d'opportunités commerciales, notamment - les gouvernements posent peu à peu les conditions de la participation des communautés à la prise de décisions relatives à de tels projets. Au plus haut degré, le principe du LCPE s'intègre directement à la législation nationale et oblige ainsi l'inclusion des préoccupations locales aux projections de développement. Cependant, les exemples suivants font état des obstacles qui s'opposent toujours au respect du droit écrit.

\section{Philippines}

Le cas des Philippines suggère que l'organisation des principes de consultation peut poser problème. Jogi Cariño, ex-commissaire de la Commission mondiale des barrages, évoque en terme de "failure to implement the "spirit" of [consultation], as distinct from "engineering consent", and formal compliance with the "letter" of the law » ${ }^{63}$. En effet, la réforme du Code minier en 1995 et la plus récente politique minière nationale posent, selon Cariño, de nouvelles menaces envers les aspirations des peuples autochtones quant au respect de leurs droits sur leurs terres. L'objectif explicite du Code minier était d'attirer plus d'investissement étranger dans le secteur minier et d'accroître la contribution de l'industrie minière à l'économie nationale ${ }^{64}$. Cette réforme a en effet attiré un important flux de nouveaux investisseurs, allant des petites compagnies sans expériences aux grands noms de l'industrie tels que Rio Tinto (Royaume-Uni et Australie), BHP (Australie), Placer Dome (Canada) et Newmount (Etats-Unis) ${ }^{65}$. Cependant, le Code minier comprend des exigences claires à l'effet que lorsqu'un développement minier est proposé sur des terres ancestrales, le LCPE des peuples autochtones concernés doit être obtenu et "sécurisé » avant que le projet n'aille de l'avant ${ }^{66}$. Pourtant, Cariño relève la répétition d'un scénario d'abus et de mésinterprétation du principe du LCPE qui couvre l'ensemble du secteur minier ${ }^{67}$.

\footnotetext{
${ }^{63}$ Supra note 10 à la p. 39. Dans le même sens, Szablowski fait état d'une interaction entre deux types de droit, parfois décrit en termes de "gap between law-in-the-books and law-in-action » en tant que défi continu. Voir Szablowski, op. cit., chapitre I.

${ }^{64}$ Supra note 10, p. 29.

${ }^{65}$ Ibid., p. 30.

${ }^{66}$ Mining Act of 1995, Republic Act 7042 par. 16: « No ancestral lands shall be opened for mining operations without the prior consent of the indigenous cultural community concerned $»$.

${ }^{67}$ Cariño, op. cit.
} 


\section{Le Libre consentement préalable et éclairé Véronique Lebuis}

Le cas du géant australien Western Mining Corporation (WMC) est intéressant. L'interaction de WMC a échoué avec les peuples B'laan concernés par son projet d'extraction de cuivre; ce fait étant bien documenté ${ }^{68}$. Devant l'opposition, WMC a également annoncé son retrait, mais a vendu ses intérêts à Indophil Holdings, contre la volonté exprimée par les B'laan. Ces derniers ont alors, avec succès, contesté en Cour suprême la légalité du Code minier de 1995 qui autorise des firmes de propriété entièrement étrangère d'exploiter les ressources minérales du pays. Ils ont également contesté la légalité du Financial and Technical Assistance Agreement (FTAA) intervenu entre le gouvernement philippin et WMC Philippines, une filiale de propriété entièrement australienne (Western Mining Corporation Holdings Limited Australia), se fondant sur la Constitution philippine de 1987 qui exige que les compagnies exploitant les ressources naturelles du pays soient contrôlées majoritairement par des intérêts philippins. La controverse vient de l'appel de cette décision par le gouvernement philippin, suite auquel la Cour a renversé sa décision initiale en affirmant que :

[t] he Constitution should be read in broad life-giving strokes. It should not be used to strangulate economic growth or to serve narrow, parochial interests...Rather it should be construed to grant the President and Congress sufficient discretion and reasonable leeway to attract foreign investment, as well as to secure for our peoples and our posterity the blessings of prosperity and peace $^{69}$.

La Cour suprême a ainsi confirmé que le Président était constitutionnellement mandaté pour conclure des accords avec des compagnies étrangères, sans égard aux pouvoirs des gouvernements locaux ni même mention des communautés autochtones, et que le Congrès avait droit de regard sur de telles ententes ${ }^{70}$. La mise en œuvre des exigences du principe du LCPE bute donc directement sur le pouvoir discrétionnaire du Président.

Pour Cariño, ce cas révèle certains abus dans la mise en œuvre du principe du LCPE, malgré les exigences explicites de la loi. Il souligne 1) le manque de respect systématique des institutions locales, des lois et pratiques coutumières, de l'opinion et des préférences des communautés; 2) le contrôle de l'information divulguée et la mauvaise interprétation de la situation locale dans les média nationaux et internationaux, en appui aux revendications des entreprises; 3) des ruses inacceptables pour influencer l'opinion locale, incluant des cas de corruption et de coercition. Par exemple, Rio Tinto, malgré l'opposition connue des communautés locales, a entrepris un travail de sondage, avec la coopération du ministère de l'Environnement et des Ressources naturelles, mais sans consultation des communautés visées.

Enfin, alors qu'un des éléments fondamentaux à l'exercice du principe du LCPE est l'accès à une information suffisante pour se forger une opinion authentique et à

\footnotetext{
${ }^{68}$ Cariño cite à cet effet Mike Boyle, «A Word of Warning: Australian Mining Corporations and the Philippine Environment », in Australian Philippines Solidarity Movement, 1996.

${ }^{69}$ Cariño, op. cit., p. 30.

${ }^{70}$ Philippines Daily Inquirer, 2 décembre 2004, p. A-1, cité dans Cariño, p. 31.
} 


\section{Le Libre consentement préalable et éclairé Véronique Lebuis}

suffisamment de temps pour évaluer et débattre de ces questions à l'intérieur des groupes concernés, Cariño affirme qu'aux Philippines, il n'existe aucun cas où l'information transmise aux communautés a été suffisante. Les consultations sont, plutôt, généralement destinées à la présentation et à la propagande de l'industrie auprès des populations affectées.

\section{Canada}

Au Canada, les relations directes entre entreprises et peuples autochtones représentent depuis peu un nouveau pôle de régulation-légitimation pour l'industrie. Ces relations se formalisent généralement dans des mécanismes qui vont du protocole d'entente (Memorandum of Understanding), non contraignant sur le plan légal, à l'entente sur les répercussions et avantages (ERA). Les ERA « are signed between mining companies and First Nation communities in Canada in order to establish formal relationships between them, to reduce the predicted impact of a mine and secure economic benefit for affected communities $"{ }^{71}$. Dans tous les cas, il s'agit de formes davantage informelles de régulation de l'investissement minier, auxquelles on a, dans la pratique, de plus en plus recours ${ }^{72}$.

En 1994, Broken Hill Proprietary Company Limited (BHP) a proposé de développer la mine d'Ekati, première mine de diamant au Canada, dans une région éloignée des Territoires du Nord-Ouest ${ }^{73}$. Cette proposition n'est pas sans inquiéter les peuples autochtones vivant dans la région concernée, considérant les multiples revendications en voie de négociations au sujet de leurs intérêts dans les terres visées ${ }^{74}$. Les consultations communautaires dans ce cas ont pris place à travers des processus et structures de prises de décision variés ${ }^{75}$. Pendant la période de négociation, plusieurs des organisations autochtones avaient leur conseiller juridique «which may have enabled them to protect

\footnotetext{
${ }^{71}$ Irene Sosa et Karyn Keenan, Impact Benefit Agreement Between Aboriginal Communities and Mining Companies: Their Use in Canada, Environmental Mining Council of British Columbia, Canadian Environmental Law Association et CooperAction: Acción Solidaria para el Desarrollo, 2001, p.1.

${ }^{72}$ Entre 1990 et 2000, dix-huit communautés autochtones de différentes provinces ont été impliquées dans le processus de négociation d'une ERA au pays. W. J. Wolfe, Socio-Economic Impact Agreements in Canada 1990-2001 Aboriginal Expectations Meet Conventional Legal, Financial and Business Practices, Prospectors and Developers Association of Canada, Toronto, 2001, p. 2.

${ }^{73}$ Voir l'étude de l'Environmental Law Institute, réalisée en collaboration avec Oxfam America, et la Sociedad peruana de Derecho ambiental, « Prior Informed Consent and Mining. Promoting the Sustainable Development of Local Comunities », 2004, à partir de la p. 16. Voir également Stefan Matiation, « Impact Benefits Agreements Between Mining Companies and Aboriginal Communities in Canada: A Model for Natural Resource Development Affecting Indigenous Groups in Latin America? », Great Plains Natural Resources Journal, vol. 7, 2002-2003, p. 204 à 232.

${ }^{74}$ Kevin O'Reilly, The BHP Independent Environmental Monitoring Agency as a Management Tool, 1998. En ligne: www.carc.org/rndtable/vbpanels.htm [dernière consultation en date du 20 avril 2008].

${ }^{75}$ Environmental Law Institute, op. cit., p. 17.
} 


\section{Le Libre consentement préalable et éclairé Véronique Lebuis}

their rights and exert greater barganing power ${ }^{76}$. L'accent a également été mis sur l'éducation des communautés affectées concernant les questions minières. Il importe à cette étape de mentionner que dans la majorité des cas où l'on juge que la formation est un critère requis pour la mise en œuvre du LCPE, les programmes sont élaborés avec l'a priori que les réticences des communautés envers un projet de développement viennent de leur manque de compréhension à l'égard des objectifs et impacts positifs et négatifs du projet $^{77}$, et ce malgré certains efforts « d'éducation interculturelle ${ }^{78}$.

Les compagnies d'extraction s'accoutument aux bénéfices qui découlent de leur engagement auprès des communautés, avant et pendant la construction d'un projet de développement :

Informal consultation between individual project managers and the heads of local communities have been replaced by procedures for systematically consulting communities. The stereotypical hard-driving mine manager operating without guidance or standards has been replaced by boardendorsed corporate policies, codified stakeholder consultation procedures, effective training, and the hiring of experienced consultation specialists. Furthermore, recognizing the legacy of the past, leading companies have been able to put together impact and benefit agreements [ERA] in concert with communities ${ }^{79}$.

Ces ERA, ayant pris forme dans les années 1990, semblent donc présenter des avenues prometteuses en termes d'employabilité, de développement économique, d'opportunités d'affaires et de protection environnementale, de même que des positions plus équitables dans certains cas, pour les communautés affectées.

\footnotetext{
${ }^{76}$ Ibid., p. 17.

${ }^{77}$ Ibid., p. 33.

${ }^{78}$ L'étude de l'Environmental Institute of Law mentionne en ce sens que «BHP made some effort to educate Agency staff by providing voluminous amount of material for the Agency's Review, as well as organizing workshops and meetings »(p.17) et souligne les conséquences d'un défaut d'éducation interculturelle des agents de liaison chargés par les compagnies de diffuser l'information dans les communautés (p. 33).

${ }^{79}$ Ethical Funds Company, Sustainability Perspectives. Winning the Social License to operate, Resource Extraction with Free, Prior and Informed Community Consent, février 2008, p. 3. [En ligne]: https://www.ethicalfunds.com/SiteCollectionDocuments/docs/FPIC.pdf, consulté le 12 septembre 2008.
} 


\section{Le Libre consentement préalable et éclairé}

Véronique Lebuis

\section{Bibliographie}

Assemblée générale des Nations Unies, Convention internationale sur l'élimination de toutes les formes de discrimination raciale, résolution $2106 \mathrm{~A}(\mathrm{XX}), 21$ décembre 1965.

Assemblée générale des Nations Unies, Déclaration sur les droits des peuples autochtones, A/RES/61/295, 13 septembre 2007.

Assemblée générale des Nations Unies, Pacte international relatif aux droits civils et politiques, 16 décembre 1966.

Assemblée générale des Nations Unies, Pacte international relatif aux droits économiques, sociaux et culturels, 16 décembre 1966.

Boisselle, Andrée, «De la consultation des peuples autochtones: Structure institutionnelle d'un dialogue appelé à renouveler la notion des droits ancestraux. Essai fondé sur la jurisprudence de la Cour suprême du Canada (1984-2004) et la théorie du droit », Mémoire de maîtrise en droit, Faculté de droit de 1'Université de Montréal, 2006.

Cariño, Jogi, «Indigenous Peoples’ Right to Free, Prior, Informed Consent: Reflections on Concept and Practice », Arizona Journal of International \& Comparative Law, vol. 22, no.1, 2005.

Colchester, Marcus et Maurizio Farhan Ferrari, « Making FPIC, Free, Prior and Informed Consent, Work: Challenges and Prospects for Indigenous Peoples », Forest Peoples Programme, juin 2007.

Comité pour l'élimination de la discrimination raciale, General Recommendation XXIII (51) concerning Indigenous Peoples, CERD/C/51/Misc.13/Rev.4., adoptée lors de la 1235 e réunion du Comité, 18 août 1997.

Commission on Human Rights, Sub-Commission on the Promotion and Protection of Human Rights, Working Group on Indigenous Populations, Twenty-third session 18-22 July 2005, E/CN.4/Sub.2/AC.4/2005/WP.1, 2005.

Cour internationale de justice, Application of the Convention on the Prevention and Punishment of the Crime of Genocide (Bosnia and Herzegovina v. Yugoslavia 


\section{Le Libre consentement préalable et éclairé \\ Véronique Lebuis}

(Serbia and Montenegro)), Separate Opinion of Judge Weeramantry, 11 juillet 1996, en ligne: http://www.icj-cij.org/docket/files/91/7360.pdf (page consultée le 21 mai 2008).

Cour Suprême du Canada, Delgamuukw c. Colombie-Britannique, 3 R.C.S. 1010, 1997.

Cour Suprême du Canada, Guérin c. La Reine, 2 R.C.S. 335, 1984.

Cour Suprême du Canada, Nation Hä̈da c. Colombie-Britannique (Ministère des forêts), 2004, 3 R.C.S. 511.

Cour Suprême du Canada, Première Nation Tlingit de Taku River c. ColombieBritannique (Directeur de l'évaluation de projet), 2004, 3 R.C.S. 550.

Cour Suprême du Canada, R. c. Gladstone, 2 R.C.S. 723, 1996.

Cour Suprême du Canada, R. c. N.T.C. Smokehouse Ltd., 2 R.C.S. 672, 1996.

Cour Suprême du Canada, R. c. Sparrow, 1 R.C.S. 1075, 1990.

Cour Suprême du Canada, R. c. Van der Peet, 2 R.C.S. 507, 1996.

Droits et démocratie, Au-delà des engagements : l'action - De la Conférence mondiale des Nations Unies sur les Droits de l'homme à la Conférence des Nations Unies sur le financement du développement, 15 mars 2002, en ligne: http://www.ddrd.ca/site/publications/index.php?id=1533\&lang=fr\&page $=4 \&$ subsection=catalog ue (page consultée le 22 mai 2008).

Duhaime, Bernard, «Évaluation des options à la portée des peuples autochtones pour veiller à l'application de la Convention 169 de l'OIT », janvier 200, en ligne : http://www.dd-rd.ca/site/publications/index.php?id=1329\&lang $=$ fr\&subsection $=\mathrm{c}$ atalogue (page consultée le 20 avril 2008).

Environmental Law Institute, «Prior Informed Consent and Mining. Promoting the Sustainable Development of Local Comunities », 2004. 


\section{Le Libre consentement préalable et éclairé}

\section{Véronique Lebuis}

Ethical Funds Company, Canadian Resource companies must adopt new standards on community engagement, communiqué de presse, Vancouver, 5 mars 2008, en ligne : www.ethicalfunds.com (page consultée le 28 avril 2008).

Ethical Funds Company, Sustainability Perspectives. Winning the Social License to operate, Resource Extraction with Free, Prior and Informed Community Consent, février 2008, en ligne, https://www.ethicalfunds.com/SiteCollectionDocuments/ docs/FPIC.pdf, (page consultée le 12 septembre 2008).

Forest Peoples Program et Tebtebba Foundation, A Submission to the Special Representative of the Secretary-General on human rights and transnational corporations and other business enterprises, 29 décembre 2006, en ligne: http://www.business-humanrights.org/Documents/Forest-Peoples-Tebtebbasubmission-to-SRSG-re-indigenous-rights-29-Dec-2006.pdf (page consultée le 22 mai 2008).

Fuller, Lon L., " Means and Ends », in K. Winston (dir.), The Principles of Social Order - Selected Essays of Lon L. Fuller, édition révisée, Hart Publishing, Oxford Portland (Oregon), 2001.

Fuller, Lon L., "Mediation - Its Forms and Functions » in K. Winston, dir., The Principles of Social Order - Selected Essays of Lon L. Fuller, édition révisée, Hart Publishing, Oxford - Portland, 2001.

Goodland, Robert, "Free, Prior and Informed Consent and the World Bank Group », Development Law \& Policy, vol. 4, 2004.

Herz, Steven, Antonio La Vina et Jonathan Sohn, « Development without conflict. The Business Case for Community Consent », World Resources Institute, mai 2007.

Inter-American Court of Human Rights, Case of the Saramaka People v. Suriname (Interpretation of the Judgment on Preliminary Objections, Merits, Reparations, and Costs), Series C No.185, 12 août 2008.

Inter-American Court of Human Rights, Case of the Saramaka People v. Suriname. Preliminary Objections, Merits, Reparations, and Costs, Serie C no. 172, 28 novembre 2007. 


\section{Le Libre consentement préalable et éclairé}

\section{Véronique Lebuis}

Inter-American Commission on Human Rights, Report No. 75/02, Case No. II.140, Mary and Carrie Dann (United States), OEA/Ser.L/V/II.116, doc. 46, 27 décembre 2002.

Inter-American Commission on Human Rights, Report No. 96/03, Maya Indigenous Communities and their Members (Case 12.053 Belize), 24 octobre 2003.

Lawrence, Sonia et Patrick Macklem, « From Consultation to Reconciliation: Aboriginal Rights and the Crown's Duty to Consult », Revue canadienne du Barreau, no. 79, 2000 .

MacKay, Fergus, «The draft World Bank Operational Policy 4.10 on Indigenous Peoples: Progress or more of the same? », Arizona Journal of International \& Comparative Law, vol. 22, 2005.

Marschke, Melissa, David Szablowski et Peter Vandergeest, «Indigenous Peoples Scoping Exercise. Synthesis Report», Working Paper 21 of the Rural Poverty and Environment Working Paper Series, Centre de Recherche en Développement international (CRDI), janvier 2007.

Matiation, Stefan, «Impact Benefits Agreements Between Mining Companies and Aboriginal Communities in Canada: A Model for Natural Resource Development Affecting Indigenous Groups in Latin America?», Great Plains Natural Resources Journal, vol. 7, 2002-2003.

O'Reilly, Kevin, The BHP Independent Environmental Monitoring Agency as a Management Tool, 1998. En ligne: www.carc.org/rndtable/vbpanels.htm (page consultée le 20 avril 2008).

Organisation internationale du Travail, Convention ( $\left.N^{\circ} 169\right)$ concernant les peuples indigènes et tribaux dans les pays indépendants.

Perreault, Anne, «Facilitating Prior Informed Consent in the Context of Genetic Resources and Traditional Knowledge », Sustainable Development Law \& Policy, vol. 4, 2004. 


\section{Le Libre consentement préalable et éclairé}

Véronique Lebuis

Rosenthal, Joshua P., «Politics, Culture and Governance in the Development of Prior Informed Consent in Indigenous Communities », Current Anthropology, vol. 47, no. 1, février 2006.

S. a., Convention sur la diversité biologique, Rio de Janeiro, 5 juin 1992, en ligne: http://www.cbd.int/doc/legal/cbd-un-fr.pdf.

S. a., Report of the International Workshop on Methodologies Regarding Free, Prior and Informed Consent and Indigenous Peoples, United Nations Permanent Forum on Indigenous Issues 2005, New York, 17-19 janvier 2005.

Sena, C. S., «An Overview of a Current Understanding of FPIC as a Methodological Issue, in Activities Relating to Indigenous Peoples: Significance and Challenges », Statement to the International Expert Workshop on Methodologies Regarding Free, Prior and Informed Consent on Indigenous Peoples, United Nations Permanent Forum on Indigenous Issues, New York, 17-19 janvier 2005.

Sosa, Irene et Karyn Keenan, Impact Benefit Agreement Between Aboriginal Communities and Mining Companies: Their Use in Canada, Environmental Mining Council of British Columbia, Canadian Environmental Law Association et CooperAction: Acción Solidaria para el Desarrollo, 2001.

St-Hilaire, Maxime, «Politique de continuité du gouvernement Charest - Vers une nouvelle génération de traités avec les autochtones? », Le Devoir, mardi 15 juillet 2003.

Szablowski, David, Transnational Law and Local Struggles. Mining, Comunities and the World Bank, Hart Publishing, Oxford et Portland, 2007.

Tebtebba Foundation, «Statement: Recent experiences and recommendations on the concept and implementation of the principle of free, prior and informed consent », 2006, en ligne, http://www.sarpn.org.za/documents/d0002040/index.php.

Wolfe, W. J., Socio-Economic Impact Agreements in Canada 1990-2001 Aboriginal Expectations Meet Conventional Legal, Financial and Business Practices, Prospectors and Developers Association of Canada, Toronto, 2001. 
Le Libre consentement préalable et éclairé

Véronique Lebuis

World Bank Group Extractive Industries, The Final Report of the Extractive Industries Review, 3 août 2004, en ligne: http://www.ifc.org/ifcext/eir.nsf/ AttachmentsByTitle/FinalResponse/SFILE/EIRFinalResponse.pdf (page consultée le 27 août 2008).

World Bank, The World Bank and Participation, Washington, DC, 1994.

World Commission on Dams, «Dams and Development, A New Framework for Decision-Making ", The Report of the World Commission on Dams, Earthscan Publications Ltd, London and Sterling, 2005. 